\title{
Influence of LOX/COX inhibitors on cell differentiation induced by all-trans retinoic acid in neuroblastoma cell lines
}

\author{
MARTINA REDOVA ${ }^{1}$, PETR CHLAPEK ${ }^{1}$, TOMAS LOJA ${ }^{1}$, KAREL ZITTERBART ${ }^{2}$, \\ MARKETA HERMANOVA ${ }^{3,4}$, JAROSLAV STERBA ${ }^{2}$ and RENATA VESELSKA ${ }^{1,2}$ \\ ${ }^{1}$ Laboratory of Tumor Biology and Genetics, Institute of Experimental Biology, School of Science, Masaryk University, Brno; \\ ${ }^{2}$ Department of Pediatric Oncology, and ${ }^{3}$ Institute of Pathology, Masaryk University and University Hospital Brno, Brno; \\ ${ }^{4}$ Institute of Pathologic Anatomy, Masaryk University and St. Anne's University Hospital, Brno, Czech Republic
}

Received August 25, 2009; Accepted October 9, 2009

DOI: 10.3892/ijmm_00000341

\begin{abstract}
We investigated the possible modulation by LOX/ COX inhibitors of all-trans retinoic acid (ATRA)-induced cell differentiation in two established neuroblastoma cell lines, SH-SY5Y and SK-N-BE(2). Caffeic acid, as an inhibitor of 5-lipoxygenase, and celecoxib, as an inhibitor of cyclooxygenase-2, were chosen for this study. The effects of the combined treatment with ATRA and LOX/COX inhibitors on neuroblastoma cells were studied using cell morphology assessment, detection of differentiation markers by immunoblotting, measurement of proliferation activity, and cell cycle analysis and apoptosis detection by flow cytometry. The results clearly demonstrated the potential of caffeic acid to enhance ATRA-induced cell differentiation, especially in the SK-N-BE(2) cell line, whereas application of celecoxib alone or with ATRA led predominantly to cytotoxic effects in both cell lines. Moreover, the higher sensitivity of the SK-N-BE(2) cell line to combined treatment with ATRA and LOX/COX inhibitors suggests that cancer stem cells are a main target for this therapeutic approach. Nevertheless, further detailed study of the phenomenon of enhanced cell differentiation by expression profiling is needed.
\end{abstract}

\section{Introduction}

Neuroblastoma is the most common extracranial malignant solid tumor in children, accounting for 6-8\% of all childhood cancers and more than $15 \%$ of pediatric cancer deaths (1). Neuroblastomas are neuroectodermal tumors arising from elements of the neural crest. The clinical behavior of neuroblastoma is heterogeneous; the likelihood of survival depends

Correspondence to: Dr Renata Veselska, Laboratory of Tumor Biology and Genetics, Institute of Experimental Biology, School of Science, Masaryk University, Brno, Kotlarska 2, 61137 Brno, Czech Republic

E-mail: veselska@sci.muni.cz

Key words: neuroblastoma, cell differentiation, retinoids, all-trans retinoic acid, caffeic acid, celecoxib on the age at diagnosis, extent of disease and tumor biology. Three different patterns are noted: life-threatening progression with fatal outcome, maturation into ganglioneuroblastoma or ganglioneuroma (even metastatic neuroblastomas have been shown to spontaneously mature to benign ganglioneuromas), or spontaneous regression (2-4).

Approximately half of the patients are diagnosed with high-risk disease, with overall survival rates less than $40 \%$ despite intensive multimodal therapy. In general, the clinical outcome of children with high-risk or relapsed neuroblastoma is poor despite the introduction of dose-intensified and highdose chemotherapy $(1,5)$. The toxicity of current treatment protocols remains significant, and there is little space to further intensify therapy. Alternative treatment strategies are thus needed in order to improve survival as well as to diminish late effects of therapy. Besides immunotherapy, which offers a much more specific and less toxic treatment than conventional therapies (6), treatment strategies that target multiple levels including tumor cells, stromal cells and angiogenesis may be of clinical benefit (7-9).

Induced differentiation of transformed cells into mature phenotypes represents a promising strategy in recent antitumor therapy (10-13). Among various inductors of differentiation, retinoids are the most frequently investigated. These derivatives of vitamin A participate in morphogenesis and differentiation processes during mammalian development. In particular, retinoic acid (RA) plays an important role in the proliferation and differentiation of many cell types and can reverse malignant growth both in vitro and in vivo (14-16).

It is evident that the biological effects of retinoic acid are mediated principally through retinoic acid receptors (RARs) and retinoid $\mathrm{X}$ receptors (RXRs). These receptors represent two subgroups of steroid and thyroid hormone receptor families that form receptor dimers, which then bind to specific response elements and regulate gene transcription (17). The role of retinoids in transcription activation machinery is particularly influenced by retinoic acid stereoisomers, i.e., all-trans retinoic acid (ATRA), 13-cis retinoic acid (13-cis RA) and 9-cis retinoic acid (9-cis RA), which are synthesized from retinaldehyde precursors. Among these, ATRA is the most abundant and thermodynamically stable form (18).

Studies comparing the activity of 13-cis RA and ATRA in neuroblastoma cell lines have demonstrated similar potency 
in terms of cell differentiation, growth arrest and regulation of gene expression (19). Furthermore, several lines of evidence suggest that 13-cis RA is likely to isomerize in vivo to ATRA and also to 9-cis RA; these are now believed to be the main biologically active forms of RA, although little is known about the mechanisms of RA isomerization in vivo $(18,20,21)$.

In the past few years, attention has also been paid to the possibility of combined induction of differentiation as well as to the modulation of differentiation by other compounds. This therapeutic approach was reported particularly for leukemia treatment; however, this strategy was also demonstrated to be effective in vitro with various types of cell lines derived from pediatric solid tumors. It has been reported that ATRAinduced differentiation in the HL-60 leukemia cell line can be further enhanced by combined application with bile acids $(22,23)$. Retinoid-induced differentiation of neuroblastoma cells may be influenced by inhibitors of enzymes that participate in the intracellular degradation pathway of retinoids, the arachidonic acid metabolic pathway, especially inhibitors of lipoxygenases (LOX) and cyclooxygenases (COX) $(24,25)$. Our study is therefore focused on the influence of arachidonic acid metabolic pathway inhibitors on ATRA-induced differentiation in neuroblastoma cell lines.

The aim of this study was to investigate a possible effect of arachidonic acid metabolic pathway inhibitors on the differentiation of SH-SY5Y and SK-N-BE(2) human neuroblastoma cell lines induced by ATRA. We hypothesized a positive modulation of the antineoplastic effect of retinoids using LOX/COX inhibitors. Caffeic acid (3,4-dihydrocinnamic acid, CA), a phenolic plant compound exhibiting non-specific anti-oxidant effects both in vivo and in vitro, was chosen as a specific 5-lipoxygenase (5-LOX) inhibitor $(25,26)$, and celecoxib (CX) was chosen as a cyclooxygenase-2 (COX-2) specific inhibitor (27-29).

\section{Materials and methods}

Cell lines. SH-SY5Y (ECACC cat. no. 94030304) and SK-N-BE(2) (ECACC cat. no. 95011815; MYCN amp.) neuroblastoma cell lines were used for this study. These cell lines were maintained in Dulbecco's modified Eagle's medium (DMEM)/Ham's F-12 medium mixture (1:1) supplemented with $20 \%$ fetal calf serum, $1 \%$ non-essential amino acids, $2 \mathrm{mM}$ L-glutamine, $100 \mathrm{U} / \mathrm{ml}$ penicillin, and $100 \mathrm{mg} / \mathrm{ml}$ streptomycin (all from PAA Laboratories $\mathrm{GmbH}$, Austria). Culture was performed under standard conditions at $37^{\circ} \mathrm{C}$ in a humidified atmosphere containing $5 \% \mathrm{CO}_{2}$. The cells were subcultured 1-2 times weekly.

Chemicals. ATRA (Sigma Chemical Co., St. Louis, MO, USA) was prepared as a stock solution at the concentration of $100 \mathrm{mM}$ in dimethyl sulfoxide (DMSO; Sigma). CA (Sigma) and CX (LKT Laboratories, Inc., St. Paul, MN, USA) were dissolved in DMSO at the concentration of 130 and $100 \mathrm{mM}$, respectively. Reagents were stored at $-20^{\circ} \mathrm{C}$ under light-free conditions.

Induction of differentiation. Stock solutions were diluted in fresh cell culture medium to obtain final concentrations of
1 and $10 \mu \mathrm{M}$ of ATRA, 13 and $52 \mu \mathrm{M}$ of CA and 10 and $50 \mu \mathrm{M}$ of CX. Concentrations of LOX/COX inhibitors were chosen on the basis of previously published data $(25,30,31)$. The final concentration of DMSO in all experiments did not exceed $0.05 \%(\mathrm{v} / \mathrm{v})$, and was found to influence neither cell proliferation nor induced differentiation. In all experiments, cells were seeded $24 \mathrm{~h}$ before the treatment, and untreated cells were used as a control.

Cell morphology. To examine cell morphology, 40,000 cells were seeded onto $8-\mathrm{cm}^{2}$ Petri dishes and allowed to attach for $24 \mathrm{~h}$ before treatment. Cell morphology was examined 2 and 7 days after ATRA and/or LOX/COX inhibitor treatment. Cell cultures were observed and recorded using an Olympus CKX41 inverted microscope in combination with an Olympus SP-350 camera.

Cell proliferation. The proliferation activity of cell populations, both treated and untreated, was measured by MTT and WST assay. For each experiment, cells were seeded in 96-well microtiter plates at a density of 10,000 cells per well and allowed to attach overnight. ATRA and/or LOX/COX inhibitors were added at the concentrations mentioned above. In order to perform the MTT assay, the culture medium was removed $48 \mathrm{~h}$ after treatment, and $220 \mu \mathrm{l}$ of DMEM/Ham's F-12 medium mixture (1:1) containing 3-[4,5-dimethylthiazol-2-yl]-2,5-diphenyltetrazolium bromide (MTT) (Sigma) at a final concentration of $455 \mu \mathrm{g} / \mathrm{ml}$ in medium was added to each well. After a 4-h incubation under standard conditions, the culture medium with MTT was replaced by $200 \mu \mathrm{l}$ of DMSO per well to solubilize the MTT product. After a 10-min shaking of the microtiter plate, the absorbance was measured at $570 \mathrm{~nm}$ using a Sunrise Absorbance Reader (Tecan, Austria $\mathrm{GmbH})$. To perform the WST assay, $10 \mu 1$ of Cell Proliferation Reagent WST-1 (Roche, Mannheim, Germany) was added to each well, and after a 4-h incubation under standard conditions and a 1-min shaking, the absorbance was measured at $450 \mathrm{~nm}$ with $620 \mathrm{~nm}$ reference wavelength using the Sunrise Absorbance Reader. Data from both MTT and WST assays were analyzed using two-way ANOVA followed by the Scheffé post-hoc test. $\mathrm{P}<0.001$ was considered significant.

Flow cytometry. To evaluate the cell cycle, 10,000 cells were seeded onto $25 \mathrm{~cm}^{2}$ Petri dishes and allowed to attach overnight. The cells were harvested 3 and 10 days after short trypsinization and stained using Vindelov's solution [0.01 M Tris, $10 \mu \mathrm{g} / \mathrm{ml}$ RNase, $50 \mu \mathrm{g} / \mathrm{ml}$ PI (all from Sigma) and $0.1 \%$ Triton X-100 (ICN Biomedicals, Irvine, CA, USA) and $1 \mathrm{mM} \mathrm{NaCl}$ ] for $30 \mathrm{~min}$ at room temperature (RT). The FACS Canto $^{\mathrm{TM}}$ II flow cytometer with I BD FASC DIVA Software (Beckton Dickinson, CA, USA) was employed to analyze the cell cycle at 3 and 10 days after treatment. Twenty thousand events per sample were evaluated using WinMDI 2.8 software (Joseph Trotter, Scripps Research Institute, La Jolla, CA, USA).

Antibodies. To perform the Western blot analysis, the following primary antibodies were used: rabbit monoclonal anti-glial fibrillary acidic protein (GFAP) antibody (cat. no. G4546, Sigma), mouse monoclonal anti-neurofilament 200 
(NF 200) antibody (cat. no. N0142, Sigma), mouse monoclonal anti-synaptophysin antibody (cat. no. S5768, Sigma), mouse monoclonal anti-neuronal nuclei (NeuN) antibody (cat. no. MAB377, Millipore, Germany) and mouse monoclonal anti-cellular retinoic acid-binding protein 1 (CRABP 1) antibody (cat. no. Ab2816, Abcam, Cambridge, UK). Secondary antibodies for immunoblotting included antimouse IgG antibody conjugated with peroxidase (cat. no. A9917, Sigma) and anti-rabbit IgG antibody conjugated with peroxidase (cat. no. A2074, Sigma). As a control, mouse monoclonal anti- $\alpha$-tubulin (cat. no. T5168, Sigma) was used.

Immunoblotting. Whole-cell extracts were loaded onto polyacrylamide gels, electrophoresed, and blotted onto polyvinylidene difluoride membranes (PVDF, Bio-Rad Laboratories $\mathrm{GmbH}$, Germany). The membranes were blocked with $5 \%$ nonfat milk in phosphate-buffered saline with $0.1 \%$ Tween 20 (PBS-T), and incubated overnight at $4{ }^{\circ} \mathrm{C}$ with selected antibodies diluted 1:1,000 in blocking solution. After rinsing with PBS-T (at least three 10-min washes), membranes were incubated with secondary antibody at 1:10,000 dilution for $45 \mathrm{~min}$ at RT. Each step was followed by at least three 10-min washes in PBS-T. ECL-Plus detection was performed according to the manufacturer's instructions (Amersham, GE Healthcare, UK).

\section{Results}

Our experiments were focused on a detailed study of the possible effect of combined treatment with ATRA and LOX/COX inhibitors (CA or CX) on neuroblastoma cells. All experiments were performed using the compounds mentioned above alone and in combinations. The course of cell differentiation was evaluated using cell morphology assessment and detection of intracellular markers by immunoblotting. The bioavailability of ATRA in the treated cell lines was studied using detection of CRABP-I protein by immunoblotting. The proliferation activity of treated cells was measured using MTT and WST assays. Changes in cell cycle and the proportion of apoptotic cells were evaluated by the use of flow cytometry.

Cell differentiation. During long-term culture, the morphology of both neuroblastoma cell lines markedly changed after induction of differentiation by ATRA, either alone or in combination with LOX/COX inhibitors in defined concentrations.

The changes in cell morphology were observed within 7 days after treatment, when the control untreated cells reached confluence. After treatment with ATRA, the first morphological changes were detectable at day 2, and they were most pronounced at day 7 (Fig. 1). ATRA applied alone (at both 1 and $10 \mu \mathrm{M}$ concentrations) or in combination with CA (at both 13 and $52 \mu \mathrm{M}$ concentrations), reduced cell proliferation and induced marked changes in cell morphology comprising the appearance of long cytoplasmic protrusions and a general neuron-like phenotype. Concerning the combination of ATRA with CA, more obvious changes in cell morphology were observed in SK-N-BE(2) cells; therefore, this cell line seems to be more sensitive to ATRA treatment in general (Fig. 1C-J). On the contrary, CA applied alone at 13 or $52 \mu \mathrm{M}$ concentrations did not affect the differentiation of both cell lines. In the presence of CA, cells proliferated rapidly to confluence (day 7 after treatment), and their morphology did not change.

Treatment of both neuroblastoma cell lines with 10 or $50 \mu \mathrm{M} \mathrm{CX}$ in combination with 1 or $10 \mu \mathrm{M}$ ATRA also led to differentiation into neuron-like phenotypes, particularly in combination with $10 \mu \mathrm{M}$ ATRA (Fig. 1S-V). However, CX applied either alone or in combination with ATRA showed a cytotoxic effect that led to an observable decrease in total cell number in culture. This phenomenon was explicitly detectable in the SH-SY5Y cell line (Fig. 1Q, R, U and V).

The course of cell differentiation in both cell lines treated with ATRA and/or LOX/COX inhibitors was also investigated in detail using immunodetection of specific differentiation markers (Fig. 2).

The induction of neuronal differentiation was confirmed by detection of neurofilament 200 (NF-200) expression. To investigate the potential ability of ATRA to induce glial differentiation, GFAP expression levels were also examined. To further verify the induction of neuronal differentiation in neuroblastoma cell lines, expression of $\mathrm{NeuN}$ as a marker of terminal differentiation of neuronal cells and synaptophysin as a neuroendocrine tumor marker was evaluated.

The expression levels of NF-200 and NeuN increased in the ATRA-treated cells of both cell lines within 7 days after treatment, confirming the potential of ATRA to induce neuronal differentiation in both neuroblastoma cell lines (Fig. 2). On the other hand, conflicting results were obtained for detection of synaptophysin: while the expression levels of synaptophysin obviously increased in both ATRA-treated and untreated SK-N-BE(2) cells in a concentration-dependent manner, the situation in the SH-SY5Y cell line was exactly the opposite (Fig. 2). In addition, the differentiation into neuronal phenotype was confirmed indirectly by complete absence of GFAP (data not shown). Furthermore, the treatment of both cell lines with LOX/COX inhibitors alone had no influence on the differentiation process since the expression of all markers was the same as in the control cells (data not shown).

The application of ATRA in combination with CA led to partly inverted results in the neuroblastoma cell lines. The enhancement of ATRA-induced neuronal differentiation by combined application with CA was confirmed in both cell lines when NF-200 was used as a marker; moreover, this enhancement by CA showed concentration dependence (Fig. 2). However, the expression of NeuN upon treatment with ATRA and CA combinations was slightly increased in SK-N-BE(2) cells and markedly decreased in SH-SY5Y cells at day 7 after treatment, especially when $1 \mu \mathrm{M}$ ATRA was used. Furthermore, a completely inverted pattern of synaptophysin expression was found in these cell lines after the same combined treatments with ATRA and CA (Fig. 2).

Detection of the same differentiation markers in both neuroblastoma cell lines after application of CX in combination with ATRA mainly corresponded to results obtained from experiments with CA/ATRA combinations. Increased levels of NF-200 after ATRA and CX combined treatment were observed in the early phases of the induced 


\section{SK-N-BE(2) cell line}
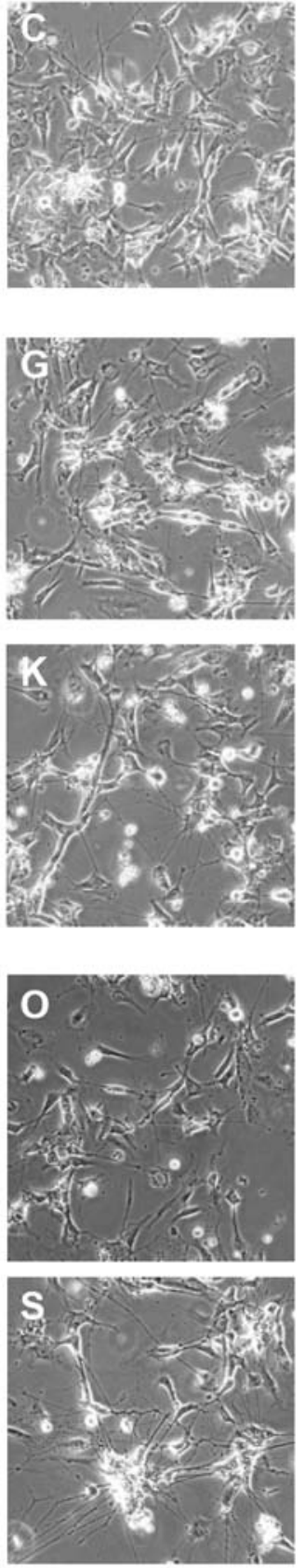

1 ATRA

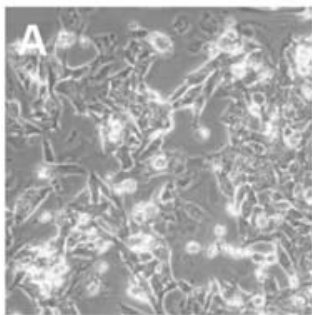

\section{SH-SY5Y cell line}
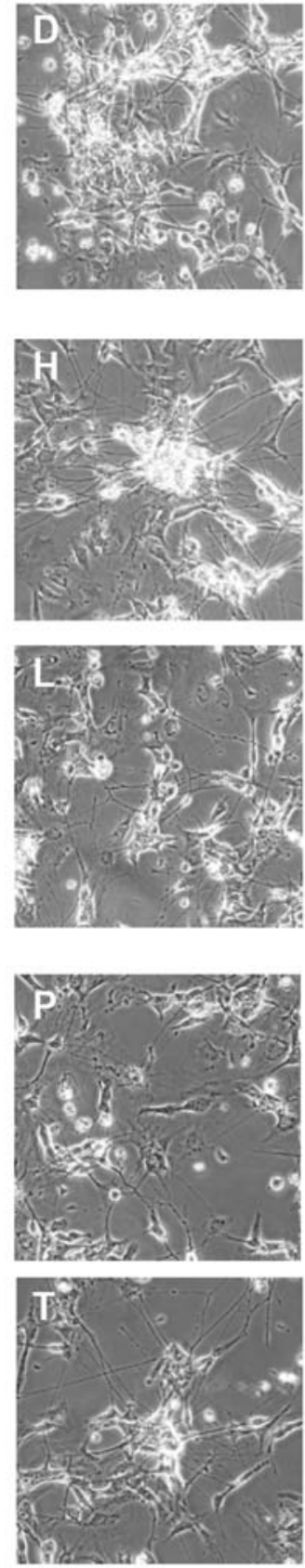

10 ATRA
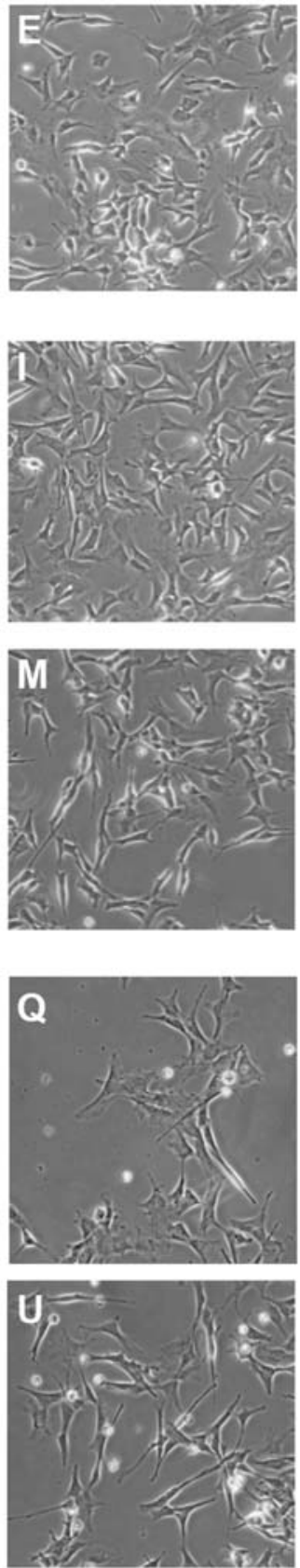

1 ATRA

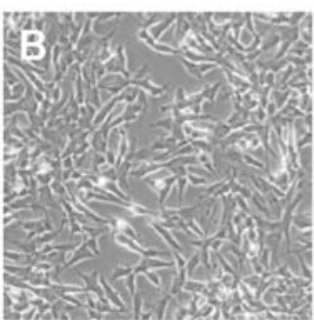

Control

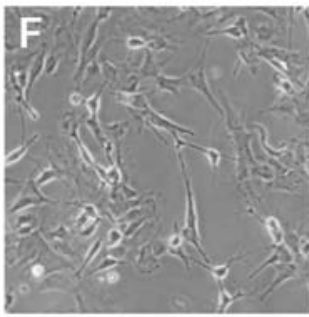

ATRA

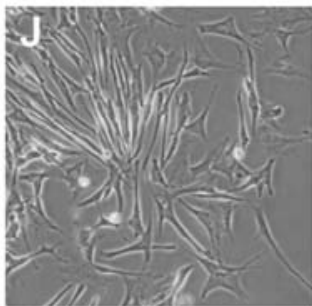

ATRA/13 CA

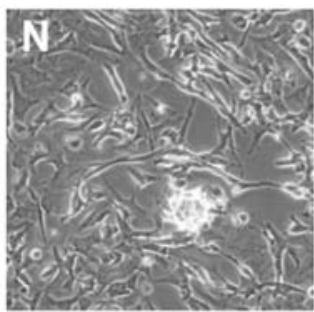

ATRA/52 CA

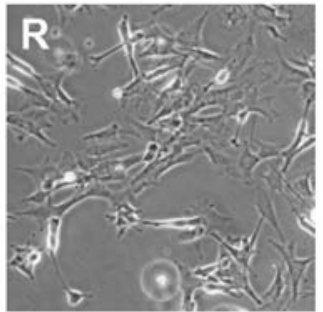

ATRA/10 CX

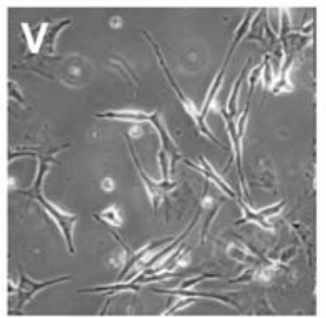

10 ATRA

Figure 1. Morphology of SK-N-BE(2) and SH-SY5Y neuroblastoma cells, both untreated (A and B) and treated with ATRA alone (C-F) in combinations with CA (G-N) or CX (O-V). ATRA was applied in concentrations of 1 or $10 \mu \mathrm{M}$ (1 ATRA, 10 ATRA); CA in concentrations of 13 and $52 \mu \mathrm{M}$ (13 CA, 52 CA), and $\mathrm{CX}$ in concentrations of 10 and $50 \mu \mathrm{M}(10 \mathrm{CX}, 50 \mathrm{CX})$. Micrographs were taken at day 7 after treatment. Original magnification, $\mathrm{x} 100$.

differentiation. However, results obtained from the late phases of the cell differentiation were more inconsistent, probably due to the cytotoxic effect of $\mathrm{CX}$ as documented in the cell morphology experiments (Figs. $1 \mathrm{O}-\mathrm{V}$ and 2). The same inverse expression pattern of NeuN in both cell lines, i.e., the increase in SK-N-BE(2) cells and decrease in SHSY5Y cells, was detected after combined treatment with ATRA and CX, similarly to the combinations with CA 

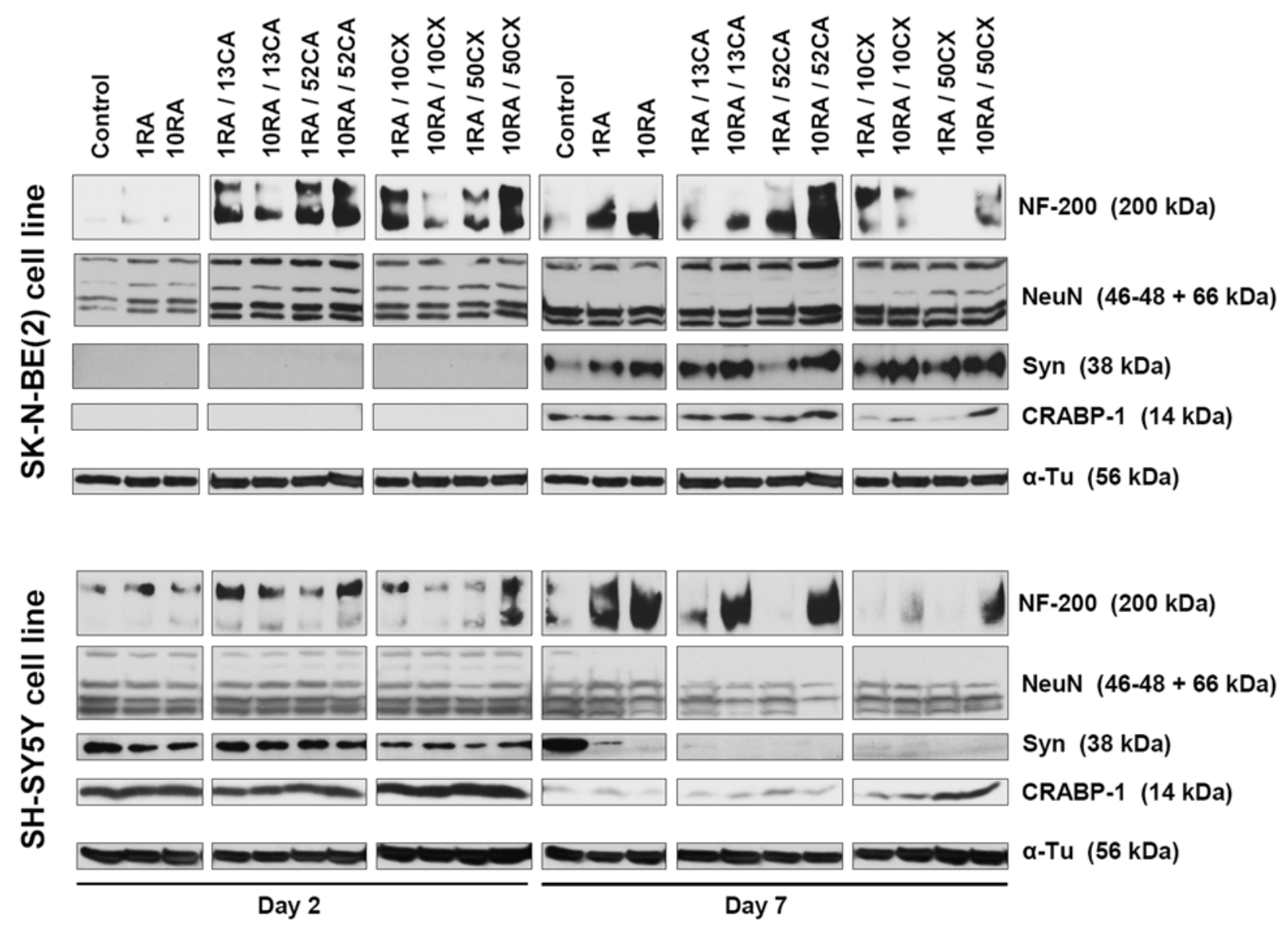

Figure 2. Western blot analysis of differentiation markers and CRABP-1 expression in SK-N-BE(2) and SH-SY5Y cells, both untreated and treated with ATRA alone or in combinations with CA or CX. ATRA was applied in concentrations of 1 or $10 \mu \mathrm{M}$ (1RA, 10RA); CA in concentrations of 13 and $52 \mu \mathrm{M}$ (13CA, 52CA), and CX in concentrations of 10 and $50 \mu \mathrm{M}(10 \mathrm{CX}, 50 \mathrm{CX})$. The immunoblotting was performed at days 2 and 7 after treatment.

described above. The same inverse pattern of synaptophysin expression was found in both cell lines after ATRA/CX combined treatment and after ATRA/CA combined treatment (Fig. 2). Moreover, changes in the spectrum of detectable NeuN bands were apparent: while the $48-\mathrm{kDa}$ band faded away in a time-dependent manner in SK-N-BE(2) cells (except for those treated with ATRA/CX), the 66-kDa band was absent in SH-SY5Y cells after 7 days of treatment.

To evaluate the intracellular activity of ATRA, expression levels of CRABP-1 were investigated simultaneously. In general, the expression patterns of CRABP-1 in both cell lines were in accordance with the inverse expression pattern observed for synaptophysin: CRABP-1 levels in SK-N-BE(2) cells increased during the cell differentiation and exhibited a slight concentration dependence after combined treatments. In the SH-SY5Y cell line, the situation was completely inverse, i.e., CRABP-1 expression markedly decreased in the late phase of cell differentiation, and only combined treatment with $50 \mu \mathrm{M} \mathrm{CX}$ resulted in maintained expression (Fig. 2).

Cell proliferation. The influence of all treatments used on the proliferation activity of both selected cell lines was examined by MTT assay for experiments with CA and combinations with ATRA, or by WST assay for CX and its combinations. The data are expressed as relative mean absorbance (ratio of treatment/control).
The application of $\mathrm{CA}$ alone, at either 13 or $52 \mu \mathrm{M}$ concentrations, generally did not affect the proliferation activity of both of these cell lines (Fig. 3A and B). Treatment with ATRA alone significantly stimulated proliferation of SK-N-BE(2) cells; however, neither stimulation nor inhibition was observed for any other treatment in this cell line (Fig. 3A). In the SH-SY5Y cell line, a slight anti-proliferative effect was detectable after combined treatment with $10 \mu \mathrm{M}$ ATRA with CA at both concentrations (Fig. 3B).

A strong cytotoxic effect of CX alone was confirmed by WST assay in both of these cell lines (Fig. 3C and D); these data are in accordance with the results obtained with cell morphology as described above. On the other hand, the application of ATRA alone at both concentrations did not significantly change the proliferation activity of SH-SY5Y cell line (Fig. 3D). However, the combined treatment led to different results: while the proliferation activity of SH-SY5Y cells was diminished by CX application independently of its combination with various concentrations of ATRA (Fig. 3D), the combined treatment with CX and ATRA reduced the inhibitory effect of $\mathrm{CX}$ in a concentration-dependent manner in SK-N-BE(2) cells (Fig. 3C).

Cell cycle and apoptosis. Finally, flow cytometry was employed to investigate in detail the influence of combined treatment with ATRA and the LOX inhibitor CA on cell 
SK-N-BE(2) cell line
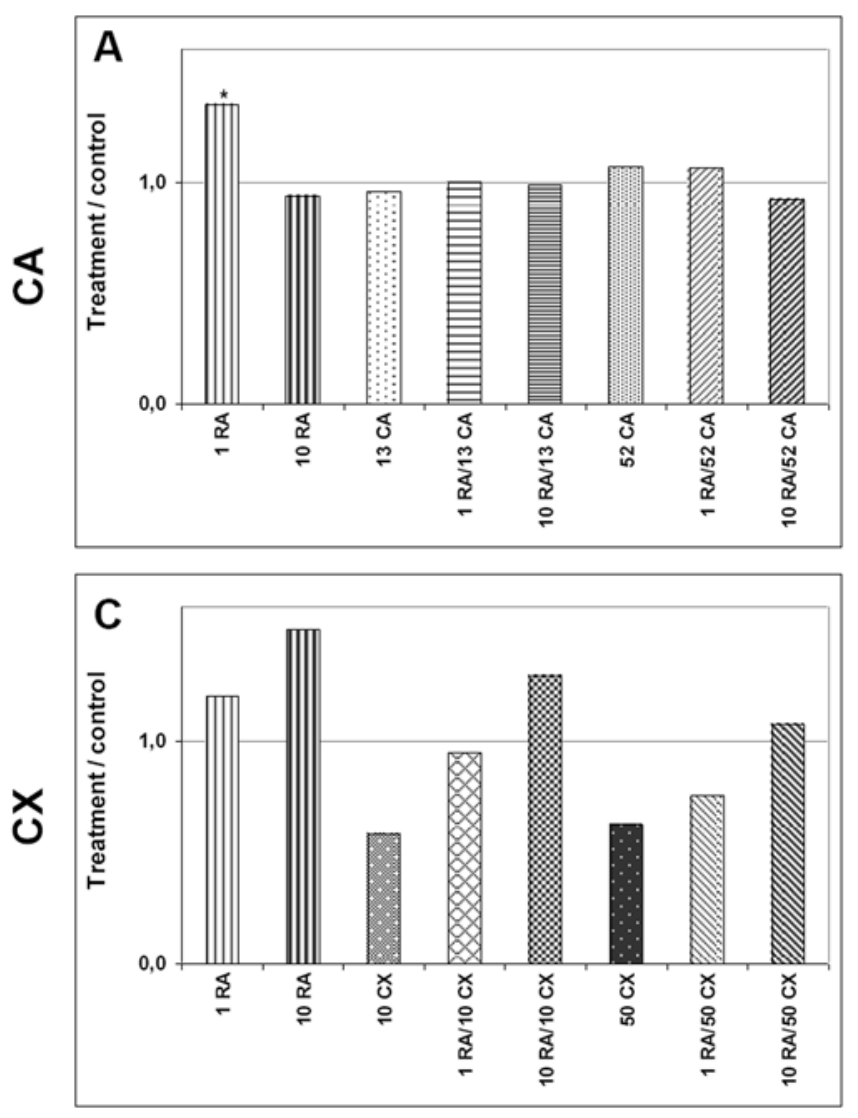

SH-SY5Y cell line
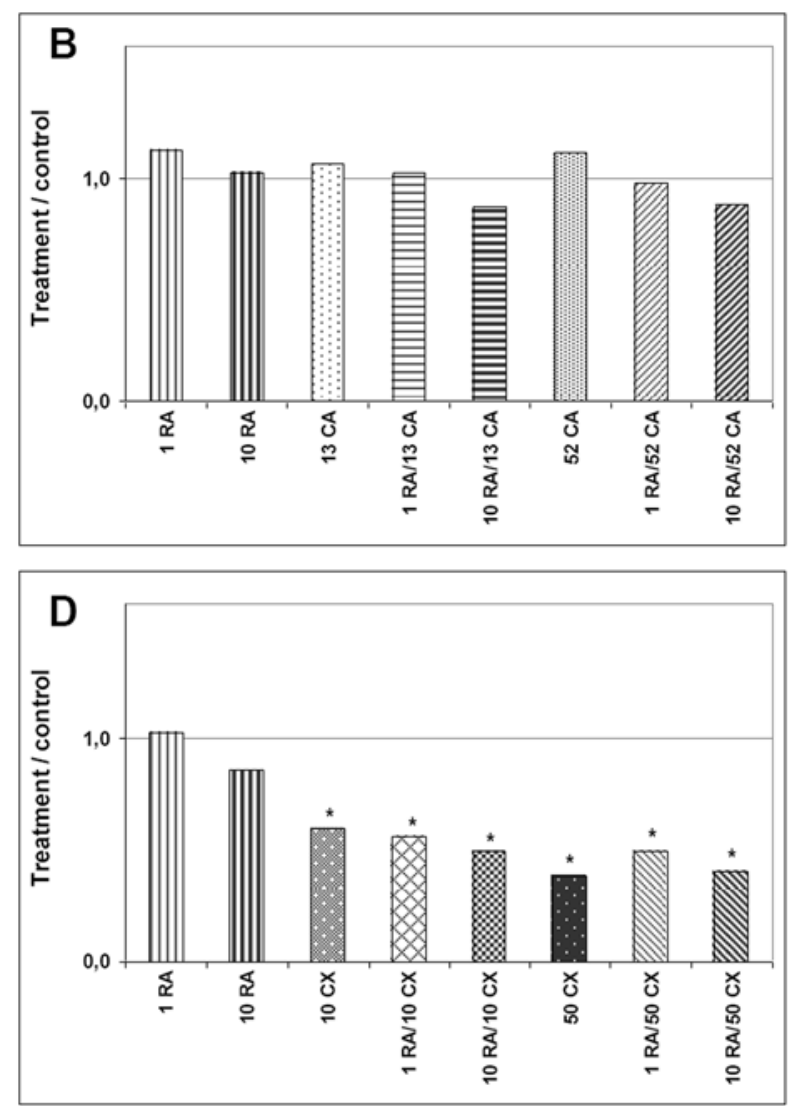

Figure 3. Proliferation activity of SK-N-BE(2) and SH-SY5Y cells treated with ATRA and/or LOX/COX inhibitors measured by MTT assay (A and B) and WST-assay (C and D), respectively. X-axis, treatment; Y-axis, relative cell number (ratio of treatment/control). Data were analyzed using two-way ANOVA followed by the Scheffé post-hoc test. "Significant difference $(\mathrm{P}<0.001)$ from the control group.

cycle and apoptosis induction in selected neuroblastoma cell lines. Application of CA alone changed neither the cell cycle course nor apoptosis frequency in SK-N-BE(2) cells (Table I); however, this compound exhibited a moderate pro-apoptotic effect accompanied by changes in the cell cycle in SH-SY5Y cells (Table II). In accordance with our other results described above, flow cytometric analysis of the DNA content confirmed higher sensitivity of the SK-N-BE(2) cell line to the combined treatment upon comparison to SH-SY5Y cells (Tables I and II, Fig. 4).

Combined application of CA with $1 \mu \mathrm{M}$ ATRA enhanced the differentiation of SK-N-BE(2) cells in a concentrationdependent manner: an increase in the number of cells in the $\mathrm{G}_{1}$-phase accompanied by a decrease in the number of cells in the $\mathrm{G}_{2} / \mathrm{M}$ phase was detected (Table I, Fig. 4A and C). In contrast, application of CA in combination with $10 \mu \mathrm{M}$ ATRA led to the shift of peak positions indicative of an increase in the number of cells in $\mathrm{G}_{2} / \mathrm{M}$ phase; in particular, an increase in the sub-diploid peak was observed which corresponded to a higher frequency of apoptosis (Table I, Fig. 4E and G).

Slight changes in the cell cycle course corresponding to induced differentiation, i.e. an increase in the number of cells in the $\mathrm{G}_{1}$-phase and a decrease in the number of cells in the $\mathrm{G}_{2} / \mathrm{M}$ phase, were observed in SH-SY5Y cells when $52 \mu \mathrm{M}$ $\mathrm{CA}$ was used for combined treatment with both concentrations of ATRA (Table I, Fig. 4D and H). Combined treatment with $13 \mu \mathrm{M}$ CA did not induce any similar effect in the same cell line (Table I, Fig. 4B and F). Furthermore, the use of $10 \mu \mathrm{M}$ ATRA alone led to the shift of the $\mathrm{G}_{2} / \mathrm{M}$ peak in the SH-SY5Y cells.

\section{Discussion}

Neuroblastoma is the most frequent extracranial solid tumor in children. It is considered to be an embryonic tumor arising from immature cells of the neural crest, probably due to genetic and molecular alterations that result in arrested cell differentiation and uncontrolled proliferation. In general, neuroblastic tumors are characterized by extreme clinical and pathological heterogeneity (32). The induced differentiation of tumor cells is regarded to be therapeutically advantageous, since more extensively differentiated neuroblastic tumors are usually associated with lower stage and better clinical outcome. Therefore, differentiating agents such as retinoic acid, which is known to induce differentiation in several tumor types including neuroblastoma, have become a part of the therapeutic arsenal (10-12,20,32).

The biological effects of RA and retinoids are generally mediated by inducible nuclear retinoid receptors (RARs and RXRs), which regulate transcription of genes with RA- 
Table I. Flow cytometric analysis of the cell cycle in SK-N-BE(2) cells. ${ }^{\mathrm{a}}$

\begin{tabular}{lcccc}
\hline Treatment & $\%<\mathrm{G}_{1}$ & $\% \mathrm{G}_{1}$ & $\% \mathrm{~S}$ & $\% \mathrm{G}_{2} / \mathrm{M}$ \\
\hline Control & 10.9 & 74.6 & 11.6 & 2.9 \\
13 CA & 13.2 & 70.5 & 10.8 & 5.5 \\
52 CA & 10.0 & 73.0 & 11.8 & 6.2 \\
1 ATRA & 35.6 & 29.0 & 21.9 & 13.5 \\
1 ATRA/13 CA & 38.0 & 25.2 & 20.9 & 15.9 \\
1 ATRA/52 CA & 33.2 & 37.7 & 14.9 & 14.2 \\
10 ATRA & 30.4 & 49.4 & 10.0 & 10.2 \\
10 ATRA/13 CA & 37.3 & 25.7 & 15.2 & 21.8 \\
10 ATRA/52 CA & 34.7 & 33.2 & 13.9 & 18.2 \\
\hline
\end{tabular}

${ }^{\text {aData }}$ from the representative experiment. Cells were analyzed at day 10 after treatment. ATRA was applied in concentrations of 1 or $10 \mu \mathrm{M}$ (1 ATRA, 10 ATRA); CA in concentrations of 13 and $52 \mu \mathrm{M}$ (13 CA, $52 \mathrm{CA})$.

Table II. Flow cytometric analysis of the cell cycle in SH-SY5Y cells. ${ }^{a}$

\begin{tabular}{lcccc}
\hline Treatment & $\%<\mathrm{G}_{1}$ & $\% \mathrm{G}_{1}$ & $\% \mathrm{~S}$ & $\% \mathrm{G}_{2} / \mathrm{M}$ \\
\hline Control & 40.1 & 25.4 & 16.5 & 18.0 \\
13 CA & 35.4 & 26.0 & 19.4 & 19.2 \\
52 CA & 37.0 & 24.9 & 19.3 & 18.8 \\
1 ATRA & 47.6 & 22.2 & 18.1 & 12.1 \\
1 ATRA/13 CA & 45.5 & 22.5 & 17.2 & 14.8 \\
1 ATRA/52 CA & 44.2 & 20.3 & 18.9 & 16.6 \\
10 ATRA & 55.4 & 20.7 & 11.9 & 12.0 \\
10 ATRA/13 CA & 54.5 & 18.5 & 15.7 & 11.3 \\
10 ATRA/52 CA & 43.7 & 19.8 & 18.4 & 16.1 \\
\hline
\end{tabular}

${ }^{\text {aD }}$ ata from the representative experiment. Cells were analyzed at day 10 after treatment. ATRA was applied in concentrations of 1 or $10 \mu \mathrm{M}$ (1 ATRA, 10 ATRA); CA in concentrations of 13 and $52 \mu \mathrm{M}$ (13 CA, $52 \mathrm{CA})$.

responsive elements $(32,33)$. The cellular retinol-binding proteins (CRBP) and cytoplasmic retinoic acid-binding proteins (CRABP-1 and CRABP-2) enhance the binding of RA to their respective receptors (34). Despite the beneficial clinical effects of treatment with RA, as mentioned above, evidence of intrinsic or acquired resistance, as well as of potential toxicity, considerably limit the use of retinoids in clinical practice. Strategies to overcome these problems consist of efforts to reduce toxicity, retain bioavailability, and develop effective combined curative regimens (35-37).

The aim of our work was to examine the possible potentiation of retinoic acid-induced differentiation of SHSY5Y and SK-N-BE(2) cells by inhibition of intracellular degradation of ATRA. Although Reynolds and co-workers
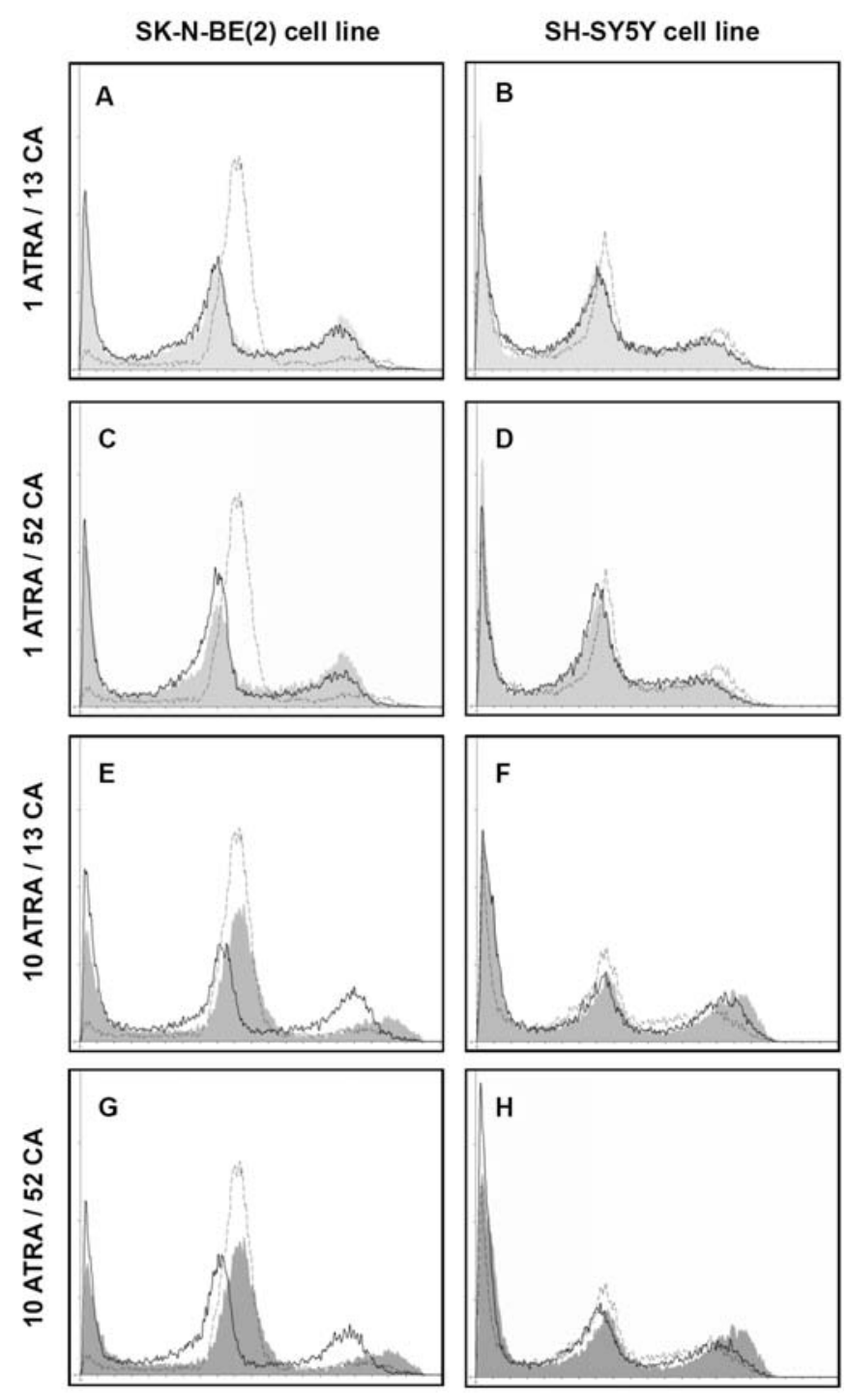

Figure 4. Representative flow cytometric analysis of the DNA content in SK-N-BE(2) and SH-SY5Y neuroblastoma cell lines at day 10 after treatment. ATRA was applied in concentrations of 1 or $10 \mu \mathrm{M}$ (1 ATRA, 10 ATRA); CA in concentrations of 13 and $52 \mu \mathrm{M}$ (13 CA, $52 \mathrm{CA})$. Histograms show results for untreated cells (open peak) overlaid with cells treated with ATRA alone (gray) and with combinations of ATRA and CA (continuous line). X-axis, logarithm of fluorescence (DNA content); Y-axis, number of events.

(20) preferred 13-cis-RA for neuroblastoma treatment, we decided to use ATRA to induce cell differentiation based on the conclusions of Armstrong and colleagues (21) that all isomers of retinoic acid undergo intracellular conversion to ATRA. CA, as an inhibitor of 5-LOX, and CX, as an inhibitor of COX-2, were chosen to study the possibility of the modulation of ATRA-induced cell differentiation in neuroblastoma cells. We investigated cell differentiation, cell proliferation, cell cycle and apoptosis in two neuroblastoma cell lines treated either with ATRA alone or with ATRA combined with LOX/ COX inhibitors.

After treatment with ATRA, marked changes in cell morphology and an increased number of cells exhibiting a neuron-like phenotype were detectable in both cell lines within 7 days; this effect was more obvious in SK-N-BE(2) cells. This observation is in accordance with results obtained with 
other methods that all confirmed higher sensitivity of SK-N$\mathrm{BE}(2)$ cells to ATRA-induced differentiation, as well as enhancement by combined treatment with CA. Analysis of cell morphology also indicated a potentiation of ATRAinduced differentiation by application of selected inhibitors in these cell lines. While treatment with inhibitors alone did not lead to the neuron-like phenotype, marked changes in cell morphology were detectable after application of ATRA alone or in combination with the inhibitors. Moreover, a strong cytotoxic effect of CX was observed in both of these cell lines.

To investigate the course of induced differentiation in neuroblastoma cell lines in detail, expression levels of specific differentiation markers were monitored. The course of neuronal differentiation in both ATRA-treated neuroblastoma cell lines was confirmed by detection of NF-200, NeuN, and synaptophysin; expression of GFAP was not detected at all. Monitoring of the expression levels of the listed markers revealed that ATRA has the potential to induce neuronal differentiation in the selected neuroblastoma cell lines, as previously described in the literature $(38,39)$. However, some ambiguous results were obtained for detection of synaptophysin expression levels: while SK-N-BE(2) cells exhibited a shift to neuronal phenotype that was time- and concentrationdependent, the situation in SH-SY5Y cells was completely inverse.

A similar discrepancy in the expression pattern of differentiation markers between these two cell lines was also observed after combined application of ATRA with inhibitors. In general, although LOX/COX inhibitors applied alone had no impact on the course of differentiation, their capability to modulate and further enhance ATRA-induced differentiation was confirmed in the SK-N-BE(2) and SH-SY5Y cell lines when NF-200 and NeuN were employed. This partial inconsistency in results for SH-SY5Y cells treated with ATRA in combination with $\mathrm{CX}$ was probably caused by the cytotoxicity of this compound, which also prevailed over cell differentiation in morphology studies.

The partially inverted pattern of results for SK-N-BE(2) and SH-SY5Y cells was probably caused by the different tumor cell type that these cell lines represent: while the SH-SY5Y cell line belongs to the N-type of neuroblastoma cell lines, SK-N-BE(2) cell line exhibits the characteristics of intermediate I-type $(40,41)$. In general, different biological features (cell shape, nuclear/cytoplasmic ratio, adherence to the substrate, differentiation potential, tumorigenicity) of $\mathrm{N}$-, I- and S-types of neuroblastoma cell lines are independent on neither $M Y C N$ amplification status nor amount of N-myc protein. The I-type was proved to exhibit a stem cell phenotype with higher differentiation potential and sensitivity to RA (41). The stem cell phenotype of SK-N-BE(2) cells was also recently confirmed by analysis of specific stem cell markers (42). Our results for SK-N-BE(2) and SH-SY5Y cell lines are in accord with these findings. Moreover, the described differences in $\mathrm{N}$ - and I-types of neuroblastoma cell lines can explain the distinctions between cell lines observed in our study.

The results from the flow cytometric analysis also demonstrated higher sensitivity of SK-N-BE(2) cells to the combined treatment with ATRA and CA. The enhancement of induced differentiation was confirmed after combined treatment with $52 \mu \mathrm{M} \mathrm{CA}$ and $1 \mu \mathrm{M}$ ATRA in SK-N-BE(2) cells, whereas a moderate pro-apoptotic effect was obvious after combined treatment of CA and $10 \mu \mathrm{M}$ ATRA in these cells, as well as in SH-SY5Y cells. The increase in the subdiploid cell fraction corresponding to a higher frequency of apoptosis was relatively surprising, since the published data suggest that ATRA does not induce apoptosis in neuroblastoma cells $(35,37,43)$.

The expression levels of CRABP-1 were investigated simultaneously to evaluate ATRA intracellular activity and availability. CRABP-1 is one of the contributors to the regulation of RA signaling pathways, sharing $75 \%$ amino acid homology and having the same molecular weight as CRABP-2 $(44,45)$. The presence or absence of different combinations of RA-binding proteins is a critical determinant of the cellular response to RA (46). The functions of CRABPs have not yet been decoded, and CRABP-1 may function differently from CRABP-2. The one feature they clearly have in common is their capability to serve as a shuttle to move RA through the aqueous cytosol to the nucleus, where RA can interact with RARs and initiate transcription of various genes. Kinetic studies of the movement of RA to RARs showed that while CRABP-1 is a passive vehicle, binding and releasing its ligand depending on concentration gradients, CRABP-2 can deliver RA to RARs in a direct collisional process (47). The detected pattern of CRABP-1 expression correlates with the observed trends in synaptophysin expression: while slight concentration- and time-dependent characteristics of CRABP-1 expression were apparent in SK-N-BE(2) cells, the results in SH-SY5Y cells were inversed. The only exception was the combined treatment with $50 \mu \mathrm{M} \mathrm{CX}$ after 7 days, which also maintained CRABP-1 expression in SH-SY5Y cells.

It has been reported that CRABP-1 and RA are inversely regulated (48). Furthermore, CRABP-1 binds RA and prevents it from entering the nucleus; in cells with low CRABP-1 expression, RA enters the nucleus (49-51). Uhrig and colleagues (52) demonstrated that up-regulation of CRABP-1 reduced the differentiation potential of SH-SY5Y cells. However, all these findings were concerned with the physiological plasma concentration of RA in humans, which is approximately $10 \mathrm{nM}$ (53). Excess exogenous RA may oversaturate the binding capacities of CRABP-1, allowing the remaining RA to bind to RARs (54). For this reason, the inverse expression patterns of CRABP-1 observable in SK-N$\mathrm{BE}(2)$ and SH-SY5Y cells may have been partly caused by the differences in cell type as mentioned above, and partly by the differential expression pattern of RA receptors across cell types.

Results of cell proliferation assays also confirmed the differences in biological behavior for these cell lines according to their sensitivity to the applied treatment. Even ATRA treatment alone showed different responses for these cell lines: while the proliferation activity of SK-N-BE(2) cells was significantly stimulated by application of ATRA alone, the same treatment in SH-SY5Y cells did not significantly increase the proliferation activity. It has been reported that treatment with ATRA results in a concentration-dependent decrease in cell proliferation (39) but other authors have 
described enhanced cell viability after application of RA (55). On the contrary, the response of both investigated cell lines to treatment with LOX/COX inhibitors applied alone was similar: CA applied alone did not affect proliferation, whereas application of $\mathrm{CX}$ alone revealed a strong cytotoxic effect. In contrast to results obtained for the course of cell differentiation, the SH-SY5Y cell line seemed to be more sensitive, exhibiting a slight anti-proliferative effect when CA was applied in combination with $10 \mu \mathrm{M}$ ATRA; a similar effect was not apparent in SK-N-BE(2) cells. However, the proliferation activity reflects the course of cell differentiation only indirectly, and this effect is probably caused by the induction of apoptosis, as apparent from the result obtained with flow cytometry in this cell line. Also, the combined treatment with CX led to different results for both of these cell lines: while the combined CX/ATRA treatment in SH-SY5Y cells decreased the proliferation activity independently of the concentrations used, in SK-N-BE(2) cells the combination of CX and ATRA reduced the inhibitory effect of CX alone in a concentration-dependent manner. Although we originally supposed that the reduction of proliferation activity in neuroblastoma cell lines is associated with the process of induced differentiation as already published by other authors $(34,38)$, the obtained results also suggest the possibility of apoptosis induction by combined treatment with ATRA and inhibitors, or a direct cytotoxic effect of CX that may be partly diminished by combined application with ATRA.

The design of our study was based on the hypothesis that inhibition of 5-LOX and/or COX-2 pathways in arachidonic acid metabolism may enhance the course of cell differentiation after treatment with ATRA in neuroblastoma cells. Our results for the SK-N-BE(2) cell line support this hypothesis, especially when CA was used as the inhibitor of 5-LOX. A similar effect of CA and its phenethyl ester on ATRA-induced cell differentiation was already shown for the HL-60 leukemia cell line $(25,56)$. This differentiation enhancement might be explained by imbalances in arachidonic acid metabolism after application of LOX/COX inhibitors. These imbalances probably increase the intracellular amount of free arachidonic acid or arachidonic acid metabolites that can potentiate the differentiation effect of RA; similar results were documented after application of exogenous arachidonic acid (24). However, this modulation of cell differentiation was less apparent when CX was used as the inhibitor of COX-2, particularly due to its cytotoxic effect, which may prevail over the possible influence of CX on ATRA-induced cell differentiation.

In conclusion, our results suggest a distinct response of SK-N-BE(2) and SH-SY5Y cells to the combined treatment. The SK-N-BE(2) cell line, which is considered to be of stem cell phenotype, was noticeably more sensitive to the enhancement of ATRA-induced cell differentiation. These results suggest that cancer stem cells, or rather tumor-initiating cells, might be the most suitable target for enhanced cell differentiation. Nevertheless, more detailed investigation of the possible mechanisms of combined induced differentiation with regard to differences in neuroblastoma cell type are needed. Therefore, our forthcoming study is focused on gene expression profiling of both of these neuroblastoma cell lines after combined treatment with ATRA and LOX/COX inhibitors.

\section{Acknowledgements}

We thank Mrs. Johana Maresova for her skillful technical assistance and Dr Michal Kyr for the statistical analysis of our data. We also thank Professor Roman Hajek for providing the flow cytometry facilities and Dr Ivana Buresova for her assistance in performing flow cytometry. This study was supported by grants IGA NR9341-3/2007, GACR 204/08/H054 and VZ MSM 0021622415.

\section{References}

1. Maris JM, Hogarty MD, Bagatell $\mathrm{R}$ and Cohn SL: Neuroblastoma. Lancet 369: 2106-2120, 2007.

2. Reynolds CP and Lemons RS: Retinoid therapy of childhood cancer. Hematol Oncol Clin North Am 15: 867-910, 2001.

3. Westermann F and Schwab M: Genetic parameters of neuroblastomas. Cancer Lett 184: 127-147, 2002.

4. Howman-Giles R, Shaw PJ, Uren RF and Chung DK: Neuroblastoma and other neuroendocrine tumors. Semin Nucl Med 37: 286-302, 2007.

5. Sterba J: Contemporary therapeutic options for children with high risk neuroblastoma. Neoplasma 49: 133-140, 2002.

6. Gray JC and Kohler JA: Immunotherapy for neuroblastoma: Turning promise into reality. Pediatr Blood Cancer 53: 931-940, 2009.

7. Stempak D, Seely D and Baruchel S: Metronomic dosing of chemotherapy: Applications in pediatric oncology. Cancer Invest 24: 432-443, 2006.

8. Sterba J, Valik D, Mudry P, Kepak T, Pavelka Z, Bajciova V, Zitterbart K, Kadlecova V and Mazanek P: Combined biodifferentiating and antiangiogenic oral metronomic therapy is feasible and effective in relapsed solid tumors in children: single-center pilot study. Onkologie 29: 308-313, 2006.

9. Andre N, Pasquier E, Verschuur A, Sterba J, Gentet JC and Rossler J: Metronomic chemotherapy in pediatric oncology: hype or hope? Arch Pediatr 16: 1158-1165, 2009.

10. Soprano DR, Qin P and Soprano KJ: Retinoic acid receptors and cancers. Annu Rev Nutr 24: 201-221, 2004.

11. Abu J, Batuwangala M, Herbert K and Symonds P: Retinoic acid and retinoid receptors: potential chemopreventive and therapeutic role in cervical cancer. Lancet Oncol 6: 712-720, 2005.

12. Coelho SM, Vaisman M and Carvalho DP: Tumour re-differentiation effect of retinoic acid: a novel therapeutic approach for advanced thyroid cancer. Curr Pharm Des 11: 2525-2531, 2005.

13. Nowak D, Stewart D and Koeffler HP: Differentation therapy of leukemia: 3 decades of development. Blood 113: 3655-3665, 2009.

14. Pasquali D, Rossi V, Bellastella G, Bellastella A and Sinisi AA: Natural and synthetic retinoids in prostate cancer. Curr Pharm Des 12: 1923-1929, 2006.

15. Garattini E, Gianni $M$ and Terao M: Retinoids as differentiating agents in oncology: a network of interactions with intracellular pathways as the basis for rational therapeutic combinations. Curr Pharm Des 13: 1375-1400, 2007.

16. Schugar RC, Robbins PD and Deasy BM: Small molecules in stem cell self-renewal and differentiation. Gene Ther 15: 126-135, 2008.

17. Knutson DC and Clagett-Dame M: atRA regulation of NEDD9, a gene involved in neurite outgrowth and cell adhesion. Arch Biochem Biophys 477: 163-174, 2008.

18. Armstrong JL, Ruiz M, Boddy AV, Redfern CP, Pearson AD and Veal GJ: Increasing the intracellular availability of all-trans retinoic acid in neuroblastoma cells. Br J Cancer 92: 696-704, 2005.

19. Veal GJ, Errington J, Redfern CP, Pearson AD and Boddy AV: Influence of isomerisation on the growth inhibitory effects and cellular activity of 13-cis and all-trans retinoic acid in neuroblastoma cells. Biochem Pharmacol 63: 207-215, 2002.

20. Reynolds CP, Matthay KK, Villablanca JG and Maurer BJ: Retinoid therapy of high-risk neuroblastoma. Cancer Lett 197: 185-192, 2003.

21. Armstrong JL, Redfern CP and Veal GJ: 13-cis retinoic acid and isomerisation in paediatric oncology - is changing shape the key to success? Biochem Pharmacol 69: 1299-1306, 2005. 
22. Zimber A, Chedeville A, Abita JP, Barbu V and Gespach C: Functional interactions between bile acids, all-trans retinoic acid, and 1,25-dihydroxy-vitamin D3 on monocytic differentiation and myeloblastin gene down-regulation in HL60 and THP-1 human leukemia cells. Cancer Res 60: 672-678, 2000 .

23. Zimber A and Gespach C: Bile acids and derivatives, their nuclear receptors FXR, PXR and ligands: role in health and disease and their therapeutic potential. Anticancer Agents Med Chem 8: 540-563, 2008

24. Hofmanová J, Kozubík A, Dusek L and Pacherník J: Inhibitors of lipoxygenase metabolism exert synergistic effects with retinoic acid on differentiation of human leukemia HL-60 cells Eur J Pharmacol 350: 273-284, 1998.

25. Veselska R, Zitterbart K, Auer J and Neradil J: Differentiation of HL-60 myeloid leukemia cells induced by all-trans retinoic acid is enhanced in combination with caffeic acid. Int $\mathrm{J}$ Mol Med 14: 305-310, 2004.

26. Vieira O, Escargueil-Blanc I, Meilhac O, Basile JP, Laranjinha J, Almeida L, Salvayre R and Nègre-Salvayre A: Effect of dietary phenolic compounds on apoptosis of human cultured endothelia cells induced by oxidized LDL. Br J Pharmacol 23: 565-573, 1998.

27. Amir M and Agarwal HK: Role of COX-2 selective inhibitors for prevention and treatment of cancer. Pharmazie 60: 563-570, 2005.

28. Shi S and Klotz U: Clinical use and pharmacological properties of selective COX-2 inhibitors. Eur J Clin Pharmacol 63: 233-252, 2008.

29. Harris RE: Cyclooxygenase-2 (cox-2) blockade in the chemoprevention of cancers of the colon, breast, prostate, and lung. Inflammopharmacology 17: 55-67, 2009

30. Nardini M, Scaccini C, Packer L and Virgili F: In vitro inhibition of the activity of phosphorylase kinase, protein kinase $\mathrm{C}$ and protein kinase $\mathrm{A}$ by caffeic acid and a procyanidin-rich pine bark (Pinus marittima) extract. Biochim Biophys Acta 1474: 219-225, 2000

31. Dandekar DS, Lopez M, Carey RI and Lokeshwar BL: Cyclooxygenase-2 inhibitor celecoxib augments chemotherapeutic drug-induced apoptosis by enhancing activation of caspase- 3 and -9 in prostate cancer cells. Int J Cancer 115 : 484-492, 2005

32. Oppenheimer O, Cheung NK and Gerald WL: The RET oncogene is a critical component of transcriptional programs associated with retinoic acid-induced differentiation in neuroblastoma. Mol Cancer Ther 6: 1300-1309, 2007.

33. Uchida D, Kawamata H, Nakashiro K, Omotehara F, Hino S Hoque MO, Begum NM, Yoshida H, Sato M and Fujimori T: Low-dose retinoic acid enhances in vitro invasiveness of human oral squamous-cell-carcinoma cell lines. Br J Cancer 85: 122-128, 2001

34. Niles RM: Signaling pathways in retinoid chemoprevention and treatment of cancer. Mutat Res 555: 81-96, 2004.

35. Ortiz MA, Bayon Y, Lopez-Hernandez FJ and Piedrafita FJ: Retinoids in combination therapies for the treatment of cancer: mechanisms and perspectives. Drug Resist Updat 5: 162-175, 2002.

36. Freemantle SJ, Spinella MJ and Dmitrovsky E: Retinoids in cancer therapy and chemoprevention: promise meets resistance. Oncogene 22: 7305-7315, 2003.

37. De los Santos M, Zambrano A and Aranda A: Combined effects of retinoic acid and histone deacetylase inhibitors on human neuroblastoma SH-SY5Y cells. Mol Cancer Ther 6: 1425-1432, 2007.

38. Voigt A, Hartmann P and Zintl F: Differentiation, proliferation and adhesion of human neuroblastoma cells after treatment with retinoic acid. Cell Adhes Commun 7: 423-440, 2000.

39. Cernaianu G, Brandmaier P, Scholz G, Ackermann OP, Alt R, Rothe K, Cross M, Witzigmann $H$ and Tröbs RB: All-trans retinoic acid arrests neuroblastoma cells in a dormant state. Subsequent nerve growth factor/brain-derived neurotrophic factor treatment adds modest benefit. J Pediatr Surg 43: 1284-1294, 2008 .
40. Ross RA, Biedler JL and Spengler BA: A role for distinct cell types in determining malignancy in human neuroblastoma cell lines and tumors. Cancer Lett 197: 35-39, 2003.

41. Walton JD, Kattan DR, Thomas SK, Spengler BA, Guo HF, Biedler JL, Cheung NKV and Ross RA: Characteristics of stem cells from human neuroblastoma cell lines and in tumors. Neoplasia 6: 838-845, 2004

42. Mahller YY, Williams JP, Baird WH, Mitton B, Grossheim J, Saeki Y, Cancelas JA, Ratner N and Cripe TP: Neuroblastoma cell lines contain pluripotent tumor initiating cells that are susceptible to a targeted oncolytic virus. PloS ONE 4: e4235, 2009.

43. Lovat PE, Ranalli M, Bernassola F, Tilby M, Malcolm AJ, Pearson AD, Piacentini M, Melino G and Redfern CP: Synergistic induction of apoptosis of neuroblastoma by fenretinide or CD437 in combination with chemotherapeutic drugs. Int $\mathrm{J}$ Cancer 88: 977-985, 2000.

44. Collins CA and Watt FM: Dynamic regulation of retinoic acidbinding proteins in developing, adult and neoplastic skin reveals roles for beta-catenin and Notch signalling. Dev Biol 324: 555-567, 2008

45. Lane MA, Xu J, Wilen EW, Sylvester R, Derguini F and Gudas LJ: LIF removal increases CRABPI and CRABPII transcripts in embryonic stem cells cultured in retinol or 4-oxoretinol. Mol Cell Endocrinol 280: 63-74, 2008

46. Schug TT, Berry DC, Shaw NS, Travis SN and Noy N: Opposing effects of retinoic acid on cell growth result from alternate activation of two different nuclear receptors. Cell 129: 723-733, 2007.

47. Dong D, Ruuska SE, Levinthal DJ and Noy N: Distinct roles for cellular retinoic acid-binding proteins I and II in regulating signaling by retinoic acid. J Biol Chem 274: 23695-23698, 1999.

48. Ruberte E, Dolle P, Chambon P and Morriss-Kay G: Retinoic acid receptors and cellular retinoid binding proteins. II. Their differential pattern of transcription during early morphogenesis in mouse embryos. Development 111: 45-60, 1991.

49. Vaessen MJ, Meijers JH, Bootsma D and Van Kessel AG: The cellular retinoic-acid-binding protein is expressed in tissues associated with retinoic-acid-induced malformations. Development 110: 371-378, 1990.

50. Dencker L, Annerwall E, Busch C and Eriksson U: Localization of specific retinoid-binding sites and expression of cellular retinoic-acid-binding protein $(C R A B P)$ in the early mouse embryo. Development 10: 343-352, 1990.

51. Dencker L, Gustafson AL, Annerwall E, Busch C and Eriksson U: Retinoid-binding proteins in craniofacial development. J Craniofac Genet Dev Biol 11: 303-314, 1991.

52. Uhrig M, Brechlin P, Jahn O, Knyazev Y, Weninger A, Busia L, Honarnejad $\mathrm{K}$, Otto $\mathrm{M}$ and Hartmann T: Upregulation of CRABP1 in human neuroblastoma cells overproducing the Alzheimer-typical Abeta42 reduces their differentiation potential. BMC Med 6: 38, 2008.

53. Kane MA, Chen N, Sparks S and Napoli JL: Quantification of endogenous retinoic acid in limited biological samples by LC/MS/MS. Biochem J 388: 363-369, 2005.

54. Morriss-Kay G: Retinoic acid and craniofacial development: molecules and morphogenesis. Bioessays 15: 9-15, 1993.

55. Kweon SM, Lee ZW, Yi SJ, Kim YM, Han JA, Paik SG and Ha SS: Protective role of tissue transglutaminase in the cell death induced by TNF-alpha in SH-SY5Y neuroblastoma cells. J Biochem Mol Biol 37: 185-191, 2004.

56. Kuo HC, Kuo WH, Lee YJ, Wang CJ and Tseng TH: Enhancement of caffeic acid phenethyl ester on all-trans retinoic acidinduced differentiation in human leukemia HL-60 cells. Toxicol Appl Pharmacol 216: 80-88, 2006. 